\title{
LA URGENTE NECESIDAD DE UNA INSTITUCIONALIDAD CIENTÍFICA PARA CHILE
}

\author{
Jorge Babul Cattan*
}

* Este artículo surgió gracias a una serie de entrevistas y conversaciones que tuvieron lugar con la periodista Jennifer Abate durante el mes de junio de 2015. 


\section{JORGE BABUL CATTTAN}

Es Licenciado en Química de la Pontificia Universidad Católica de Chile y Doctor en Bioquímica de la Universidad de Iowa, Estados Unidos. Es profesor titular de la Universidad de Chile en el Departamento de Biología de la Facultad de Ciencias. Actualmente se desempeña como Director del Programa Académico de Bachillerato del mismo plantel. 


\section{LA URGENTE NECESIDAD DE UNA INSTITUCIONALIDAD CIENTÍFICA PARA CHILE}

Para quienes hemos desarrollado una carrera de larga data en el mundo de la ciencia, la falta de planificación estatal respecto a la investigación científica que necesita Chile para su desarrollo no es un tema nuevo. Sin embargo, se trata de una realidad que hoy, de la mano del colapso del sistema de Becas Chile, que junto al programa de becas nacionales ha logrado formar un gran número de investigadores especializados, pero sin asegurar condiciones mínimas para la inserción de los mismos en la academia o las empresas productivas chilenas, se está volviendo visible para otros actores sociales. Ya no cabe duda: Chile necesita urgentemente de una institucionalidad científica que no solo le permita planificar ordenadamente el trabajo que debe realizar en esta área, sino una que, quizás más crucialmente, le permita determinar qué ciencia necesita nuestro país para alcanzar su pleno desarrollo.

\section{LA GÉNESIS DE BECAS CHILE}

Corría el año 2008 y Chile acababa de ingresar a la Organización para la Cooperación y el Desarrollo Económico (OCDE), un selecto grupo integrado por los países más desarrollados del mundo. Por supuesto se trataba de una noticia alegre, pero también implicaba un desafío mayúsculo: la comparación de los indicadores nacionales con los de los países de este grupo resultaba, en muchos casos, desoladora, e implicaría un esfuerzo enorme intentar alcanzarlos. Puntualmente, el Estado chileno encendió las alarmas debido a la distancia entre nuestro país y otros como Dinamarca o Finlandia en cuanto a la brecha de escolaridad. Chile estaba visiblemente atrasado en relación con esas naciones y necesitaba de muchos doctorados y posdoctorados capaces de liderar el progreso en todas las áreas. Así fue como nació el programa Becas Chile, administrado por la Comisión Nacional de Investigación Científica y Tecnológica (CONICYT), que vino a reemplazar las becas Presidente de la República y que, entre otras cosas, amplió la oferta para los profesionales que querían perfeccionarse en universidades extranjeras.

En el período entre 2008 y 2012 se asignaron más de cinco mil becas de magíster y doctorado en el exterior, tres mil más de las que se habían entregado en los cinco años previos. Bien en cuanto a los indicadores de escolaridad, pero 
este aumento también hizo que se acrecentara un problema soterrado que muchos conocemos desde hace años y que hoy se está volviendo visible para muchos otros y particularmente para quienes intentan forjar una carrera en el mundo de la ciencia: estos profesionales no tienen lugar para insertarse laboralmente, debido a que no existió ni existe en Chile una planificación relacionada con el retorno de estos profesionales a las aulas, laboratorios o empresas chilenas.

La génesis de este problema es clara: el programa Becas Chile se hizo sin la participación de las universidades, que son las instituciones y unidades académicas que tienen los programas de doctorado. Esto provocó que al inicio se concedieran muchas becas y que la gente saliera, literalmente, a "pasear" a universidades de poco prestigio y escasa exigencia. Resulta difícil determinar en qué se pensó en ese momento, pero da la impresión de que nunca existió una planificación clara respecto de qué se iba a hacer con todos esos doctorados. A esto se suma la necesidad de muchos científicos de seguir estudios de posgrado especializados para poder destacar dentro de sus determinadas áreas, lo que los hizo presa de la ingenuidad de pensar que este país los iba a esperar con los brazos abiertos y solo ha acrecentado su frustración, sobre todo entre los científicos más jóvenes.

CONICYT tiene aproximadamente cuatro mil becarios de doctorado, una buena parte de estos cursando estudios en el extranjero; sin embargo, aún no hemos logrado diseñar un plan apropiado para insertarlos en las universidades y en las empresas.

Pronto se graduará un número de estudiantes que iguala el número de profesores con el grado de doctor que trabaja en las universidades y que se ha incorporado a ellas a lo largo de más de 40 años. Estas, por cierto, necesitan académicos de alto nivel, no solo por la investigación que realizarían, sino también por la importancia que tienen en la docencia y en la formación de profesionales en general. Sin embargo, de nada sirve aumentar este número si no se trabaja, al mismo tiempo, en la planificación estratégica de lo que Chile quiere hacer con ellos.

Existe consenso en cuanto a la importancia de la investigación y el desarrollo y la baja inversión de nuestro país en esta área, en especial la del sector privado. Para aumentar la inversión de las empresas se plantea el establecimiento de un puente entre ellas y las universidades o centros de investigación. Sin embargo, el Estado no ha logrado definir una institucionalidad que conduzca apropiadamente este proceso. Más aún, se afirma que existe falta de conducción y coordinación entre los ministerios de Educación y Economía, responsables de las políticas en estas materias, y se ha planteado como solución posible la creación de un ministerio de Ciencia y Tecnología.

¿Quién hace las proposiciones y decide las políticas? ¿Participan todos los actores involucrados? ¿Quién manda en ciencia? Actualmente, tanto CONICYT 
como el Consejo de Innovación se sobreponen en su misión, pues ambos están destinados a asesorar a la Presidenta de la República y promover y fomentar la ciencia y tecnología en Chile.

¿A qué se debe tanto desorden y descoordinación? La conclusión es clara: no existen en Chile los canales apropiados para que la comunidad científica participe de la toma de decisiones respecto al desarrollo de la ciencia en el país. Si bien se ha insistido en este punto, los esfuerzos desplegados por el Consejo de Sociedades Científicas respecto a la necesidad de generar una verdadera institucionalidad para la ciencia, con un rango similar al ministerial, no han tenido la recepción merecida.

En vez de impulsar la dispersión de iniciativas, Chile necesita ordenar la construcción de una institucionalidad integral, con rango ministerial, que se ocupe de todas estas materias, incorporando, indispensablemente, la participación de todos los actores involucrados.

En este sentido, el caso de Becas Chile es solo uno más de los que demuestran las graves consecuencias de políticas de Estado incapaces de ocuparse del desarrollo del país en sentido amplio. Otro muy notable es la escasez de universidades dedicadas a la investigación en Chile. Estas se han constituido en uno de los pilares esenciales del desarrollo de los países, tanto por la investigación que realizan en la búsqueda de nuevos conocimientos como en la formación de personas con conocimiento avanzado en programas de doctorado en las diversas disciplinas.

Sin embargo, tanto en el mundo como en nuestro país no más del 10\% de las universidades son de este tipo.

En cuanto a la investigación científica, los estudios del grupo SCImago indican que en los últimos años Chile ocupa el lugar 45 (de 225 países) en publicaciones por año, produciendo el 7,5\% de estas en América Latina (AL) y el 0,28\% del mundo.

En este ranking figuran 19 universidades chilenas y se destacan la Universidad de Chile (UCh) en el lugar 425 ( 1 en Chile y 12 en AL), la Pontificia Universidad Católica de Chile (PUC) en el lugar 589 (2 en Chile y 18 en AL) y la Universidad de Concepción (UCo) en el lugar 912 (3 en Chile y 32 en AL).

En el período 2007-2011, el 65\% de las cerca de 30 mil publicaciones nacionales correspondieron a esas tres universidades: UCh, 26\%; PUC, $24 \%$ y UCo, $15 \%$. Por otra parte, esas mismas universidades, en el mismo período, obtuvieron el 56\% de los Proyectos FONDECYT Regular: UCh, 27\%; PUC, 20\% y Uco, 9\%.

Igualmente, en el último concurso nacional de becas de doctorado de CONICYT estas universidades obtuvieron el 70\% de las becas: UCh, 33\%; PUC, 23\% y UCo $14 \%$, y graduaron aproximadamente $70 \%$ de los doctores del país: UCh, 30\%; PUC $26 \%$ y UCo $15 \%$.

Por cierto que uno podría extender el análisis de la investigación que se hace en el país y compararla con la de países más desarrollados. En ese caso la situación es 
alarmante, tanto en la inversión en ciencia (solo $0,44 \%$ de nuestro PIB) como en el número de investigadores (seis mil jornadas completas equivalentes) o la falta de una institucionalidad y una política de desarrollo estables.

$\mathrm{Al}$ menos dos aspectos son claves dentro de este análisis. Por una parte es claro que necesitamos más universidades de investigación en el país, especialmente en las regiones, ya que la mayor parte de nuestra ciencia se concentra en la Región Metropolitana (aproximadamente el 55\%). Sin embargo, por otra, también necesitamos apoyar fuertemente a las ya existentes. ¿Cómo lograr estos objetivos? Las tres principales universidades descritas más que duplican en sus presupuestos a todo lo que el país gasta anualmente en ciencia y tecnología, pero solo reciben un aporte fiscal de alrededor del 10\% de sus presupuestos. El apoyo al financiamiento de estas universidades constituye tanto un deber como un desafío para el próximo gobierno y debe tener toda la atención posible de nuestros candidatos a la presidencia.

Según la Real Academia Española, "conocimiento" significa "acción y efecto de conocer; entendimiento, inteligencia, razón natural; noción, ciencia, sabiduría". Para muchos, como para el profesor español Federico Mayor Z., conocimiento indica más frecuentemente sabiduría que saber: comportamiento sensato, lúcido, sereno. Así, es necesario, por el bien de nuestro país, que cuando emprendamos en serio las acciones para lograr la llamada economía basada en el conocimiento, tengamos muy en cuenta este significado. Requerimos una economía basada en puntos de referencia éticos y no conducida por los propósitos del mercado; una economía que incite a la creatividad y que promueva la expresión de la diversidad cultural. La mirada debe ser de futuro, vale decir, en nuestros jóvenes. Necesitamos un desarrollo donde no se propague el desencanto y la marginación entre aquellos que deben convertirse en los protagonistas de los cambios que el país necesita.

\section{COMISIÓN ASESORA PRESIDENCIAL}

Una luz de esperanza se asomó este año con la creación de la Comisión Asesora Presidencial Ciencia para el Desarrollo de Chile, presentada a finales de enero y compuesta por 35 expertos que van desde biólogos a economistas, pasando por un sociólogo, un historiador y una sicóloga, para pensar el tipo de ciencia que necesita Chile para su desarrollo y hacer recomendaciones a la Presidenta de la República.

La presentación de este comité no pudo ser más auspiciosa. En esa fecha, la Presidenta Michelle Bachelet anunció que se modificaría el financiamiento de centros de investigación como los Institutos Milenio, Basales y FONDAP, quitando la limitación de financiamiento público actual, que actualmente acaba a los 10 años. Al mismo tiempo, aseguró que se presentaría un proyecto de ley capaz de otorgar institucionalidad por ley (y no por decreto, como ocurre hoy) al Consejo Nacional 
de Innovación, y que se reactivaría la Comisión Interministerial de Innovación, a la que ella asistiría al menos una vez al año.

Se trata de anuncios importantes que han reactivado nuestras esperanzas y alimentado el trabajo que esta comisión ha acometido arduamente. Pero a pesar de nuestro esfuerzo y entusiasmo, nos hace falta ser reconocidos y validados por la ciudadanía. Si le preguntamos a cualquier persona cuántos científicos somos y qué hacemos, no va a tener idea. Una vez dije una frase que me cambió la vida: "vamos a dar el gran salto hacia el desarrollo cuando sea la ciudadanía y no la comunidad científica la que pida más ciencia”. Eso significa tener una sociedad educada, que sepa qué es y para qué sirve la ciencia. Que cuando yo le cuente un experimento con peras y manzanas ojalá disfrute tanto como con un gol de Alexis Sánchez, por poner un ejemplo ahora, que acaba de terminar la Copa América. Ese es uno de los puntos de esta comisión, no se trata solo de crear el ministerio.

El tiempo apremia. Estamos trabajando desde marzo en esto y nos corresponde hacer el trabajo que no se ha hecho en 40 años, es decir, determinar cómo insertar la ciencia como pilar fundamental del desarrollo del país. En ese sentido, nuestro trabajo no termina cuando entreguemos el informe de la propuesta, esto termina cuando consigamos todo. Y ese todo implica conseguir que haya una institucionalidad apropiada para la ciencia, que podría ser un ministerio donde participen las personas que tienen que ver con nuestro desarrollo científico. En este momento no hay una organización donde se junten los científicos con los políticos, con los empresarios a pensar en Chile, en los problemas que tiene y en cómo resolverlos. A lo largo de mi vida he aprendido que las políticas no vienen de arriba, vienen de abajo para arriba. Nosotros, que estamos en la base, tenemos que decir cómo tiene que ser esta nueva política.

\section{TODOS POR LA CIENCIA}

En esta lucha por dotar a Chile de una institucionalidad científica adecuada, no solo el Estado como estructura, sino que todos los actores sociales tienen una enorme responsabilidad. Por una parte está el rol de las universidades estatales, como la Universidad de Chile. Evidentemente, todas las universidades tienen que tener su visión, pero hay visiones que son particulares. Lo ejemplifico: una vez, cuando era Director de Pregrado en la Universidad de Chile, me topé con un muchacho durante el proceso de matrícula y me contó que era judío y que por esa razón no podía sino estudiar en la Universidad de Chile, haciendo hincapié en que no podría haber cursado sus estudios en otra universidad que, a pesar de su calidad, no lo acogería de igual manera debido a su carácter confesional. 
Sin duda que hay que ser respetuoso de las visiones que cada universidad tiene, pero la misión de los planteles estatales en la formación del conocimiento, el que incluye las ciencias, las ciencias sociales, las humanidades y más, es entregar libertad a sus estudiantes, dejarlos ser. El Estado tiene como misión hacer cosas que nadie más va a hacer porque no son rentables. Son mil cosas y es una obligación del Estado preocuparse no solo de garantizar la diversidad, sino también de promover la diversidad, de incitar que seamos diferentes con el fin de alcanzar el desarrollo de los distintos aspectos del quehacer humano. Esto, la diversidad, el responderse preguntas nuevas, es esencial para el desarrollo científico y en esta línea las universidades del Estado son fundamentales. En el Estado caben todos, esa es la gracia.

Por otra parte necesitamos, los mismos científicos, encontrar la forma de comunicar la ciencia a la población de una manera que le haga sentido. Nosotros tenemos que cumplir con una labor muy importante, que es hacerle ver a las personas que lo que está pasando tiene que ver con nosotros. Algo así como decir: "Mire, señora, usted tiene esa cafetera y usted la enchufa y gracias a mí se calienta el café, porque nosotros en este país tenemos una buena parte de la energía eléctrica que proviene de la energía solar y tenemos unas plantas de energía solar en el norte y para hacer esas placas que reciben la luz y la transforman en energía se necesitan todos estos materiales. Algunos de esos materiales están en la tierra, pero no es llegar y sacarlos; tiene que haber gente que los estudie y yo le enseño a los profesionales que van y sacan esos materiales, que trabajan en las empresas y que hacen las placas que reciben la energía que le llega a usted".

Si entendiéramos eso, si la gente entendiera eso con claridad, no tendríamos que explicar la necesidad de que todos los proyectos de ley pasaran por un comité científico. En la actualidad hay un grupo pequeño en el Senado, unos pocos senadores que pertenecen a la comisión de ciencia y tecnología y un poco más numerosa en el caso de los diputados, que están preocupados de este tema. Estamos trabajando con ellos y nos hemos dado cuenta de cosas interesantes. Por ejemplo, el presidente de la comisión, que es el senador Guido Girardi, encargó un estudio comparativo en los distintos ministerios con ítems que tienen que ver con ciencia y tecnología, de manera de poder aumentar el presupuesto destinado a ellos. La conclusión fue alarmante: estamos por debajo de los números que había el año 2013.

Es para solucionar estos problemas y para comunicar la ciencia de manera que interese a la gente que necesitamos más científicos. Somos muy pocos y hacemos un trabajo muy arduo. Cada uno tiene un proyecto científico, hace docencia, investigación y tiene su familia. Yo tengo que trabajar fuera de hora para hacer todo lo que hago. La gente a veces piensa que lo único que nos interesa es publicar en revistas indexadas, pero es lo que tenemos que hacer para permanecer vigentes y 
mantener nuestro prestigio. Este país sería distinto si tuviéramos más científicos. Se dice que somos entre ocho mil y 10 mil por el número de proyectos que están funcionando. Habría que, por lo menos, multiplicarlos por 10. 